\title{
Difference in the emetic control among highly emetogenic chemotherapy regimens: Implementation for appropriate use of aprepitant
}

\author{
SHINYA AOKI ${ }^{1}$, HIROTOSHI IIHARA ${ }^{2}$, MINAKO NISHIGAKI ${ }^{2}$, YOSHINORI IMANISHI ${ }^{2}$, \\ KEITA YAMAUCHI $^{2}$, MASASHI ISHIHARA ${ }^{2}$, KIYOYUKI KITAICHI ${ }^{2}$ and YOSHINORI ITOH $^{2}$ \\ ${ }^{1}$ Laboratory of Clinical Pharmacy, Gifu Pharmaceutical University, Gifu 501-1196; \\ ${ }^{2}$ Department of Pharmacy, Gifu University Hospital, Gifu 501-1194, Japan
}

Received May 14, 2012; Accepted July 24, 2012

DOI: $10.3892 / \mathrm{mco} .2012 .15$

\begin{abstract}
Although antiemetic medication based on the emetogenicity of the cancer chemotherapy regimen is recommended, emetic control varies even among highly emetogenic chemotherapy (HEC). In the present study, we retrospectively investigated the rates of emetic control by a combination of granisetron, $5-\mathrm{HT}_{3}$ antagonist and dexamethasone in various HEC regimens, including 5 single-day chemotherapy regimens such as gemcitabine/cisplatin (GEM/CDDP), epirubicin/cyclophosphamide(EPI/CPA),pemetrexedorvinorelbine/cisplatin (PEM or VNR/CDDP), doxorubicin/bleomycin/ vinblastine/dacarbazine (ABVd) and rituximab/doxorubicin/ cyclophosphamide/vincristine/prendisolone (R-CHOP21), and 2 multiple-day chemotherapy regimens such as 5-fluorouracil/cisplatin (5-FU/CDDP) and bleomycin/etoposide/ cisplatin (BEP). Complete response (no emesis, no rescue treatment) during the overall period (days 1-5) was assessed as the primary endpoint. Chemotherapy-induced nausea and vomiting was well-controlled (complete response $>70 \%$ ) in GEM/CDDP and R-CHOP21, but not in other regimens. The effect of a triple antiemetic medication including aprepitant (APR) was subsequently examined in patients receiving EPI/ CPA and 5-FU/CDDP. Complete response was significantly improved in patients receiving 5-FU/CDDP but not in those receiving EPI/CPA, although the complete protection from vomiting significantly increased in both cases. Of note, the administration of APR for 5 days, but not for 3 days, was required to completely block the incidence of vomiting during
\end{abstract}

Correspondence to: Dr Shinya Aoki, Laboratory of Clinical Pharmacy, Gifu Pharmaceutical University, 1-25-4 Daigaku-nishi, Gifu 501-1196, Japan

E-mail: aokis@gifu-pu.ac.jp

Key words: highly emetogenic chemotherapy, single-day chemotherapy regimen, multiple-day chemotherapy regimen, chemotherapy-induced nausea and vomiting, complete response, aprepitant the 7 days of the observation period in patients receiving 5-FU/CDDP. These findings suggest that APR should be used appropriately based on the emetogenicity of HEC regimens.

\section{Introduction}

Chemotherapy-induced nausea and vomiting (CINV) is a frequent distressing side effect that impairs patient's quality of life and decreases medication adherence (1-3). CINV comprises the acute event that occurs within $24 \mathrm{~h}$ of chemotherapy, the delayed event that appears after $24 \mathrm{~h}$ persisting for several days, and the anticipatory symptom that develops prior to chemotherapy, particularly in patients who experienced CINV in the previous course (4). In the clinical practice guidelines for the prevention of CINV documented by the Multinational Association of Supportive Care in Cancer (MASCC) (5), the American Society of Clinical Oncology (ASCO) (6) and the National Comprehensive Cancer Network (NCCN) (7), anticancer agents are classified into four risk categories based on the emetogenicity: high emetic risk (HEC), moderate emetic risk (MEC), low emetic risk and minimal emetic risk. Prophylactic medication against CINV was recommended based on the evidence of the eligible clinical studies. For example, triple combination therapy, including neurokinin $\mathrm{NK}_{1}$ receptor antagonist, 5-HT antagonist (granisetron) and dexamethasone (DEX), is recommended to prevent CINV associated with HEC regimens.

Aprepitant (APR) is a selective $\mathrm{NK}_{1}$ antagonist for the substance $P$ in the central nervous system. Several eligible clinical trials evaluating the antiemetic effect of APR in patients receiving high doses of cisplatin $\left(\geq 70 \mathrm{mg} / \mathrm{m}^{2}\right)$ or anthracycline/ cyclophosphamide demonstrated that APR combined with the standard antiemetic medication, comprising $5-\mathrm{HT}_{3}$ antagonist and DEX, significantly improved complete response (no emesis and no rescue treatment) compared to the standard antiemetic therapy (8-11). APR is shown to be effective against acute as well as delayed emesis, in which the efficacy is independent of gender $(10,12)$. However, involvement of the $\mathrm{NK}_{1}$-sensitive mechanism may vary among different chemotherapeutic regimens, even in the HEC regimens. In the present study, we retrospectively analyzed the rates of emetic control by a 
combination therapy, comprising $5-\mathrm{HT}_{3}$ antagonist and DEX, in patients receiving 5 single-day treatment HEC regimens such as gemcitabin/cisplatin (GEM/CDDP), epirubicin/cyclophosphamide (EPI/CPA), pemetrexed or vinorelbine/cisplatin (PEM or VNR/CDDP), doxorubicin/bleomycin/vinblastine/ dacarbazine $(\mathrm{ABVd})$ and rituximab/doxorubicin/cyclophosphamide/vincristine/prednisolone (R-CHOP21), as well as 2 multiple-day chemotherapy regimens, such as 5 -fluorouracil/ cisplatin (5-FU/CDDP) and bleomycin/etoposide/cisplatin (BEP). Subsequently, the effect of a triple combination antiemetic therapy using APR, 5- $\mathrm{HT}_{3}$ antagonist and DEX was prospectively investigated in breast cancer patients receiving EPI/CPA and head-and-neck cancer patients who underwent a 5-FU/CDDP regimen.

\section{Patients and methods}

Study design. This study comprises a retrospective chart review of the emetic control by a combination therapy consisting of 5- $\mathrm{HT}_{3}$ antagonist and DEX in several HEC regimens and a non-randomized prospective study evaluating the antiemetic effect of a triple combination therapy of APR, 5- $\mathrm{HT}_{3}$ antagonist and DEX in single- as well as multiple-day HEC regimens. The present study was carried out in accordance with the guidelines for the care for human study adopted by the Ethics Committee of the Gifu Graduate School of Medicine (Gifu, Japan), and approved by the Japanese government (no. 22-156 of the Institutional Review Board).

Patients. Patients who underwent the HEC regimen for the first time (first course) at Gifu University Hospital (Gifu, Japan) between April 14, 2009 and November 18, 2011, were the subject of the present study. The exclusion criteria were age, $<18$ years; patients receiving emetogenic drugs, such as opioid analgesics; patients receiving previous chemotherapy; and those with organic disorders accompanied by nausea and vomiting.

HEC regimens. As shown in Table I, HEC regimens were 5 single- and 2 multiple-day chemotherapy regimens, including GEM/CDDP (GEM $1,000 \mathrm{mg} / \mathrm{m}^{2}$, days 1,8 and 15 and CDDP $70 \mathrm{mg} / \mathrm{m}^{2}$, day 1 , every 28 days) for bladder cancer (13); R-CHOP21 (rituximab $375 \mathrm{mg} / \mathrm{m}^{2}$, day 1; doxorubicin $50 \mathrm{mg} / \mathrm{m}^{2}$, day 3 ; CPA $750 \mathrm{mg} / \mathrm{m}^{2}$, day 3 ; vincristine $1.4 \mathrm{mg} / \mathrm{m}^{2}$, day 3 and prednisolone $100 \mathrm{mg} /$ body, days $3-7$, every 21 days) for malignant B-cell lymphoma (14); EPI/ CPA (EPI $90 \mathrm{mg} / \mathrm{m}^{2}$, day 1 and CPA $600 \mathrm{mg} / \mathrm{m}^{2}$, day 1 , every 21 days) for breast cancer (15); PEM/CDDP (PEM $500 \mathrm{mg} / \mathrm{m}^{2}$, day 1 and CDDP $75 \mathrm{mg} / \mathrm{m}^{2}$, day 1 , every 21 days) or VNR/CDDP (VNR $25 \mathrm{mg} / \mathrm{m}^{2}$, days 1 and 8 and CDDP $80 \mathrm{mg} / \mathrm{m}^{2}$, day 1 , every 21 days) for non-small cell lung cancer $(16,17)$ and $\mathrm{ABVd}$ (doxorubicin $25 \mathrm{mg} / \mathrm{m}^{2}$, day 1 ; bleomycin $10 \mathrm{mg} / \mathrm{m}^{2}$, day 1 ; vinblastine $6 \mathrm{mg} / \mathrm{m}^{2}$, day 1 and dacarbazine $250 \mathrm{mg} / \mathrm{m}^{2}$, day 1 , every 14 days) for Hodgkin's lymphoma (18) for single-day regimens; 5-FU/CDDP (5-FU $800 \mathrm{mg} / \mathrm{m}^{2}$, days $1-5$ and CDDP $80 \mathrm{mg} / \mathrm{m}^{2}$, day 1 , every 21 days) for head-and-neck cancer (19); and BEP (bleomycin $30 \mathrm{mg} /$ body, days 1,8 and 15 ; etoposide $100 \mathrm{mg} / \mathrm{m}^{2}$, days $1-5$ and CDDP $20 \mathrm{mg} / \mathrm{m}^{2}$, days $1-5$, every 21 days) for testicular cancer (20).
Antiemetic medication. Prior to the addition of APR, a combination therapy, including granisetron ( $3 \mathrm{mg}$, day 1 ) and DEX (20 mg intravenously on day 1 and $8 \mathrm{mg}$ orally on days $2-4$ ), was a common antiemetic medication against HEC regimens. Dopamine $\mathrm{D}_{2}$ antagonists such as prochlorperazine and metoclopramide, antipsychotic agents, including olanzapine, antihistaminic agents such as diphenhydramine and histamine $\mathrm{H}_{2}$ blockers, including famotidine, were used as the breakthrough treatment for CINV. In a set of studies, where the antiemetic effect of APR was evaluated in patients receiving EPI/CPA or 5-FU/CDDP, APR was administered orally at $125 \mathrm{mg}$ on day 1 and $80 \mathrm{mg}$ on days 2 and 3 or on days 2-5 in addition to a combination therapy using granisetron and DEX.

Emetic control. Unless otherwise indicated, the incidence of CINV during the 5 days of the observation period was checked from the pharmaceutical record where clinical pharmacists recorded the symptoms and severity of adverse drug events during daily monitoring in pharmaceutical care practices. In the case of 5-FU/CDDP, the observation period was extended from 5 to 7 days). Complete response (no vomiting, no rescue treatment) during the overall (0-5 or 0-7 days) period was a primary endpoint. Complete response during acute $(0-24 \mathrm{~h}$ after chemotherapy) and delayed (2-5 or 2-7 days) periods, and complete protection from vomiting were assessed as secondary endpoints.

Statistical analysis. Data were analyzed using the Statistics Program for Social Sciences (SPSS X, version 11) for Windows (SPSS, Inc., Chicago, IL, USA). The rate of complete response or complete protection from vomiting was compared prior to and following the addition of APR to the standard combination antiemetic therapy and statistically evaluated by Fisher's exact probability test. $\mathrm{P}<0.05$ was considered to indicate a statistically significant difference.

\section{Results}

Comparison of the rates of emetic control response by a combination of granisetron and DEX among various HEC regimens. The efficacy of combination therapy using granisetron and DEX was evaluated in patients who received the first course of HEC regimens, excluding R-CHOP21 in which DEX was not administered. Although acute CINV was well-controlled in most cases, the complete response during the delayed period varied among various chemotherapy regimens (Fig. 1). A good overall complete response rate was observed in the GEM/ $\operatorname{CDDP}(71.4 \%, \mathrm{n}=14)$ and $\mathrm{R}-\mathrm{CHOP} 21(73.7 \%, \mathrm{n}=19)$ regimens, however, the rate was extremely low in $\operatorname{BEP}(12.5 \%, \mathrm{n}=16)$ and ABVd $(7.7 \%, \mathrm{n}=13)$. The response rate was moderate in EPI/ CPA $(50.0 \%, n=20)$, PEM or VNR/CDDP $(46.2 \%, n=13)$ and 5 -FU/CDDP $(27.3 \%, \mathrm{n}=11)$.

Effect of a triple antiemetic treatment using APR, granisetron and DEX. Subsequently, the effect of adding APR to the standard combination therapy was investigated in patients receiving EPI/CPA or 5-FU/CDDP. As shown in Fig. 2A, APR caused a slight, but not significant, improvement of complete response in EPI/CPA, in which the complete response 


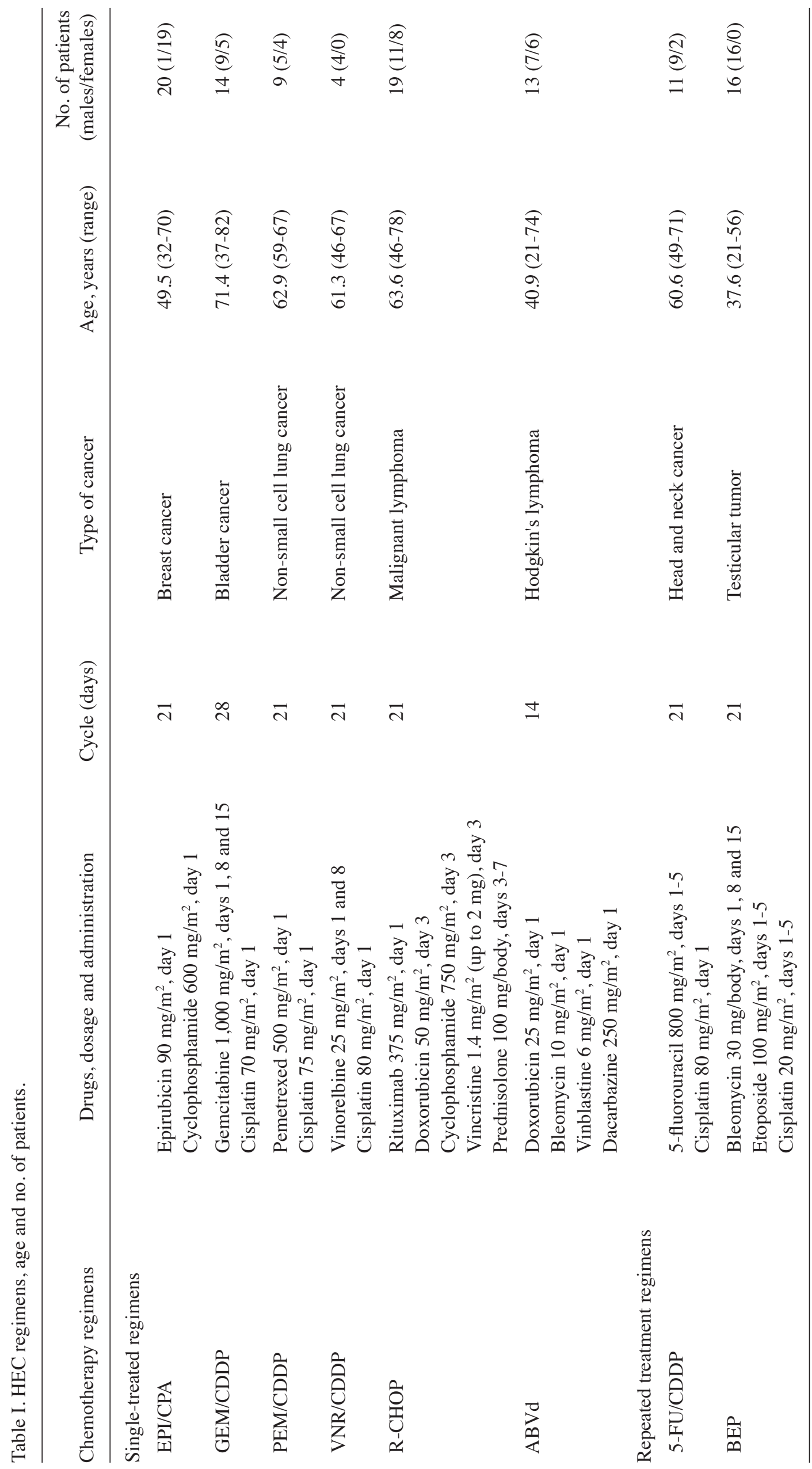


A Single-treatment regimen
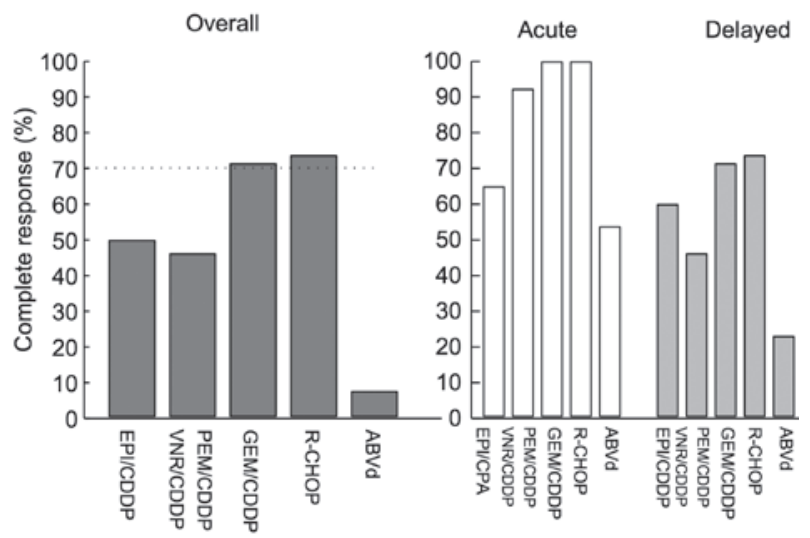

\section{B Repeated treatment regimen}
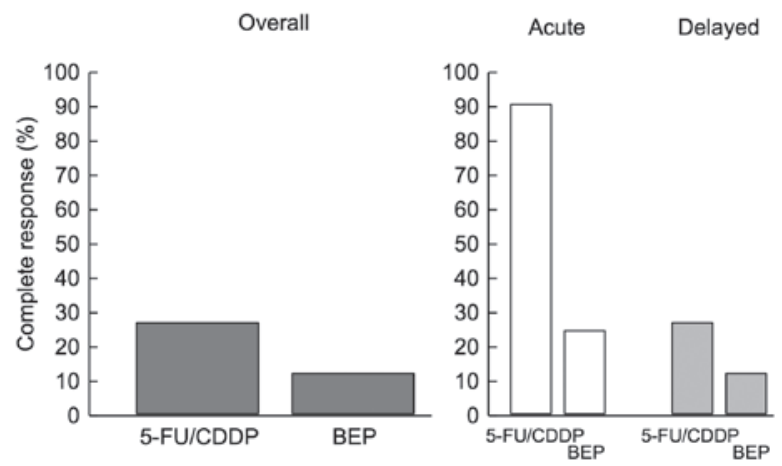

Figure 1. Comparison of the rates of emetic control response by a combination of granisetron and DEX among various HEC regimens. Chemotherapy regimens included: (A) 5 single-treatment regimens such as EPI/CPA $(n=20)$, PEM or VNR/CDDP ( $n=13)$, GEM/CDDP $(n=14)$, ABVd $(n=13)$ and R-CHOP21 (n=19), and (B) 2 multiple-day chemotherapy regimens, including 5-FU/CDDP $(\mathrm{n}=11)$ and BEP $(\mathrm{n}=16)$. All patients, except for those receiving R-CHOP21, were administered intravenous granisetron and DEX $(20 \mathrm{mg})$ on day 1 prior to chemotherapy, followed by oral DEX $(8 \mathrm{mg})$ on days 2-4 for the prevention of CINV. In patients receiving R-CHOP21, only granisetron was injected on day 1 .
A

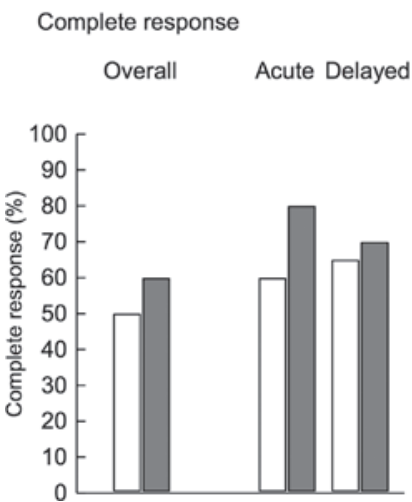

B

Complete protection from vomiting Overall Acute Delayed

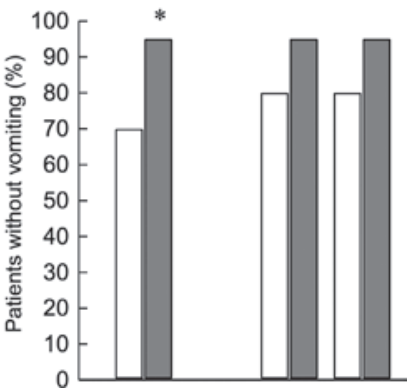

Figure 2. Effect of a triple antiemetic treatment using APR, granisetron and DEX on the (A) control response and (B) complete protection from vomiting in patients receiving the first course of EPI/CPA regimen. Patients were all administered intravenous granisetron ( $3 \mathrm{mg})$ and DEX $(12 \mathrm{mg})$ and oral APR $(125 \mathrm{mg})$ on day 1 prior to chemotherapy, followed by oral DEX $(8 \mathrm{mg})$ on days 2-4 and APR $(80 \mathrm{mg})$ on days 2 and 3 . The rate of complete response or complete protection from vomiting was assessed during acute (day 1), delayed (days $2-5$ ) and overall periods (days $1-5$ ). ${ }^{*} \mathrm{P}<0.05$ by Fisher's exact probability test.
A

Complete response

Overall

Acute Delayed

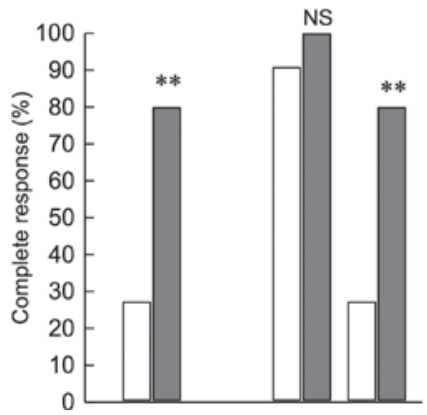

B

With APR $(n=20)$

Complete protection from vomiting

Overall Acute Delayed

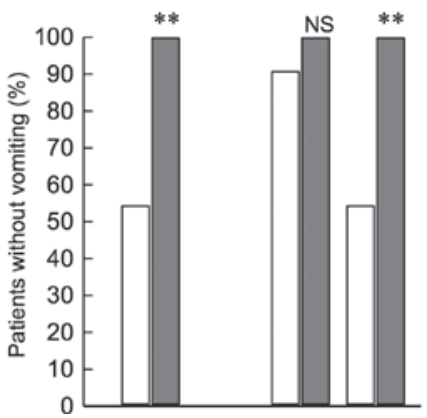

Figure 3. Effect of a triple combination therapy using APR, granisetron and DEX on (A) the control response and (B) complete protection from vomiting in patients receiving the first course of 5-FU/CDDP regimen. In the APRtreated group, intravenous granisetron $(3 \mathrm{mg})$ and DEX $(12 \mathrm{mg})$ and oral APR (125 mg) were administered prior to chemotherapy, followed by oral DEX $(8 \mathrm{mg}$ ) on days $2-5$ and APR $(80 \mathrm{mg})$ on days $2-3$ or $2-5$, while in the control group, intravenous granisetron $(3 \mathrm{mg})$ and DEX $(20 \mathrm{mg})$ were injected prior to chemotherapy, and oral DEX (8 mg) was administered on days 2-5. The rate of complete response or complete protection from vomiting was assessed during the acute (day 1), delayed (days 2-5) and overall periods (days 1-5). ${ }^{* * *} \mathrm{P}<0.01$ by Fisher's exact probability test.

during acute, delayed and overall periods was improved by $20(\mathrm{P}=0.301), 5(\mathrm{P}=1.000)$ and $10 \%(\mathrm{P}=0.751)$, respectively, although the complete protection from vomiting during the overall period was significantly $(\mathrm{P}<0.05)$ improved from 70.0 to $95.0 \%$ (Fig. 2B).

By contrast, the complete response and complete protection from vomiting during the overall (days 1-5) period were significantly improved in the 5-FU/CDDP regimen (from 27.3 to $80.0 \%, \mathrm{P}<0.01$, for complete response; from 54.5 to $100 \%$, $\mathrm{P}<0.01$, for complete protection from vomiting), in which the relative risk for overall complete response was 2.933 [95\% confidence intervals (CI)], 1.090-7.891) (Fig. 3).

However, the control of CINV decreased on days 6 and 7 in patients administered with APR for 3 days (Fig. 4A), resulting in a marked impairment of the overall complete response (Fig. 4C), when the observation period was extended to 7 days $(40.0 \%$ during the 7 -day period vs. $60.0 \%$ during the 5-day period). The administration of APR $(80 \mathrm{mg} / \mathrm{day})$ for the remaining 2 days (days 2-5) completely eradicated the incidence of vomiting (Fig. 4B), in which the complete protection from vomiting was increased, although this increase ( $\mathrm{P}=0.0526$ ), from 60.0 to $100 \%$ (Fig. $4 \mathrm{D}$ ), was not significant. The overall (days 1-7) complete response showed an improvement from 40.0 to $66.7 \%$ ( $\mathrm{P}=0.347$ ) (Fig. 4C).

\section{Discussion}

HEC regimens examined in the present study consisted of cisplatin-based chemotherapy, dacarbazine-based chemotherapy and a combination chemotherapy of anthracycline and cyclophosphamide such as EPI/CPA and R-CHOP21. The antiemetic effect of a combination therapy using granisetron and DEX markedly varied among chemotherapy regimens. A good overall complete response was observed in the GEM/CDDP 
A

Aprepitant for 3 days $(\mathbf{n}=5)$

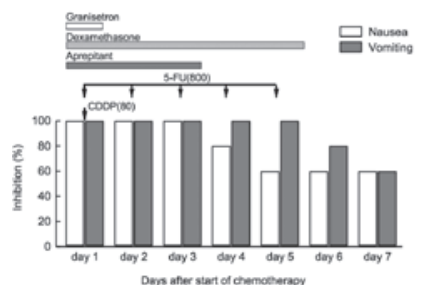

C

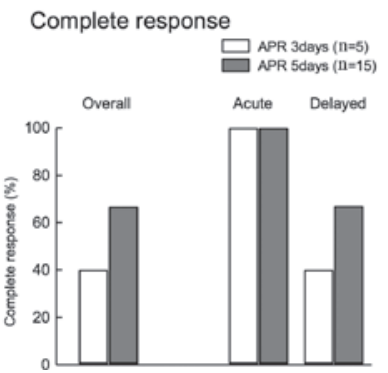

B

Aprepitant for 5 days $(\mathbf{n}=15)$

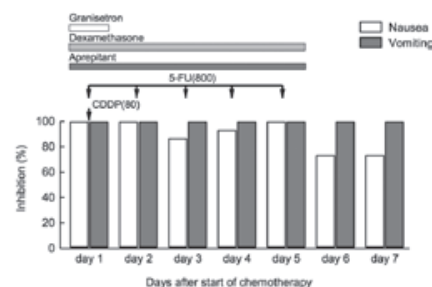

D

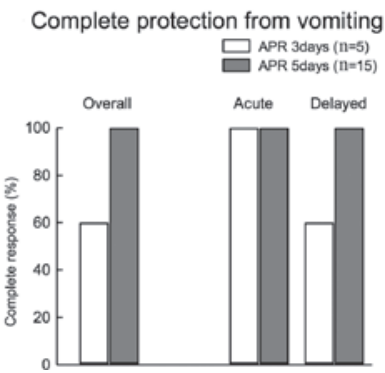

Figure 4. Comparison of time course in the complete protection from nausea and vomiting following the administration of APR for (A) 3 or (B) 5 days after the initiation of chemotherapy. Comparison of the effects of 3-and 5-day APR treatment schedule on the (C) complete response and (D) complete protection from vomiting in patients receiving the first course of 5-FU/CDDP regimen for head-and-neck cancer (C). APR was administered at $125 \mathrm{mg}$ on day 1 and at $80 \mathrm{mg}$ on (A, C and D) days 2-3 or (B, C and D) 2-5. Patients were administered intravenous granisetron (3 mg) and DEX (12 mg) and APR $(125 \mathrm{mg})$ on day 1 prior to chemotherapy, followed by DEX $(8 \mathrm{mg})$ on days 2-5 and APR (80 mg) on days $2-3$ or 2-5.

(71.4\%) and R-CHOP21 (73.7\%) regimens, a moderate response was evident in EPI/CPA $(50.0 \%), \mathrm{PEM} / \mathrm{CDDP}$ or VNR/CDDP (46.2\%) and 5-FU/CDDP (27.3\%) regimens, but the response was extremely poor in BEP (12.5\%) and ABVd (7.7\%) regimens. Our data on the complete response in the R-CHOP21 regimen were generally consistent with the data reported by Vitolo et al (21). In that study, most of the patients (79\%) exhibited no gastrointestinal adverse reactions, including CINV. By contrast, the rate of complete response in the GEM/CDDP regimen observed in the present study was much higher than that reported by Dogliotti et al (22), in which the incidence of CINV was $74.5 \%$, indicating that the complete protection from CINV was assumed to be $25.5 \%$. Although we were not able to elucidate the difference between findings of that study and those of this study, the discrepancy may be due to the fact that CINV was monitored only in the first course of the chemotherapy in our study, whereas up to six courses of chemotherapy were examined in the study by Dogliotti et al (22).

Although several clinical practice guidelines for the prevention of CINV documented by MASCC (5), ASCO (6), and NCCN (7) recommend the use of a triple combination such as $\mathrm{NK}_{1}$ antagonist, 5-HT 3 antagonist and DEX for the prevention of CINV associated with HEC regimens, a combination of two drugs such as 5- $\mathrm{HT}_{3}$ antagonist and DEX was considered to be satisfactory for the prophylaxis of CINV in GEM/ CDDP for bladder cancer and R-CHOP21 for malignant B-cell lymphoma on the first course of chemotherapy. However, the

addition of APR should be considered to prevent CINV effectively in other chemotherapy regimens, including EPI/CPA for breast cancer, PEM or VNR/CDDP for lung cancer, 5-FU/ CDDP for head-and-neck cancer, BEP for testicular tumor and ABVd for Hodgkin's lymphoma.

Therefore, we evaluated the efficacy of triple combination antiemetic medication, including APR, granisetron and DEX, in a single-day HEC regimen such as EPI/CPA and multipleday chemotherapy regimens such as 5-FU/CDDP. APR caused a slight, but not significant, increase in the complete response rate in breast cancer patients receiving EPI/CPA, although the rate of complete protection from vomiting was significantly $(\mathrm{P}<0.05)$ elevated, after APR was added, from 70.0 to $95.0 \%$ (relative risk, 1.357; 95\% CI, 1.001-1.839). A slight but significant preventive antiemetic effect of APR has also been reported by Warr et al (23) in breast cancer patients receiving anthracycline/cyclophosphamide combination chemotherapy. Those authors showed that the overall complete response is enhanced by $8.3 \%$ (from 42.5 to $50.8 \%$ ) following the addition of APR to the combination therapy of ondansetron and DEX.

In contrast to these findings, APR resulted in a marked and significant $(\mathrm{P}<0.01)$ improvement of the overall complete response from 27.3 to $80.0 \%$ in the 5-FU/CDDP regimen, when the observation period was set to 5 days. The incidence of vomiting 5 days after chemotherapy was completely blocked by the administration of APR for 3 or 5 days $(\mathrm{P}<0.01)$. Adding APR to a combination antiemetic therapy has been proven to improve the complete response by approximately $15-20 \%$ in patients receiving cisplatin-based chemotherapy $(8,10,24)$. Chawla et al $(25)$ reported a more marked improvement of the overall complete response, in which the rate is elevated from 43.7 to $71.0 \%$ after the addition of APR to a combination therapy in patients receiving cisplatin $\left(\geq 70 \mathrm{mg} / \mathrm{m}^{2}\right)$-based chemotherapy.

However, the control of nausea and vomiting was impaired at 6 and 7 days after chemotherapy, resulting in a reduction of the overall complete response $(40.0 \%)$, when the observation period was extended to 7 days. It was notable that the addition of APR for an additional 2 days (days 1-5) completely blocked the incidence of vomiting during the 7-day period of time and the overall (days 1-7) complete response was improved, though not significantly ( $\mathrm{P}=0.347)$, to $66.7 \%$ as compared to the 3-day APR treatment regimen (40.0\%). Therefore, it is likely that an extended administration of APR is required for the prevention of CINV in patients receiving the 5-FU/CDDP regimen for head-and-neck cancer. The safety and efficacy of extended APR treatment in the multiple-day HEC and MEC regimens were also reported by Jordan et al (26), although those authors did not compare the complete response between the multiple-day APR and the standard 3-day APR treatment regimens.

In conclusion, the present data on the differences in the rates of emetic control by a combination therapy using $5-\mathrm{HT}_{3}$ antagonist and DEX suggest that APR is not required for an initial course of a few HEC regimens such as GEM/CDDP and R-CHOP21. However, administration of APR for 5 days, instead of for 3 days, was more effective in preventing CINV in a multiple-day chemotherapy regimen such as 5-FU/CDDP. Therefore, appropriate use of APR should be carried out depending on the emetogenicity of each HEC regimen. 


\section{Acknowledgements}

This study was supported by a Grant-in-Aid for Scientific Research from the Ministry of Education, Culture, Sports, Science and Technology of Japan (no. 22590134 for Y.Itoh and no. 21928002 for H.I.).

\section{References}

1. Bloechl-Daum B, Deuson RR, Mavros P, Hansen M and Herrstedt J: Delayed nausea and vomiting continue to reduce patients' quality of life after highly and moderately emetogenic chemotherapy despite antiemetic treatment. J Clin Oncol 24: 4472-4478, 2006.

2. Lachaine J, Yelle L, Kaizer L, Dufour A, Hopkins S and Deuson R: Chemotherapy-induced emesis: quality of life and economic impact in the context of current practice in Canada. Support Cancer Ther 2: 181-187, 2005.

3. Sun CC, Bodurka DC, Weaver CB, et al: Rankings and symptom assessments of side effects from chemotherapy: insights from experienced patients with ovarian cancer. Support Care Cancer 13: 219-227, 2005.

4. Roscoe JA, Morrow GR, Aapro MS, Molassiotis A and Olver I: Anticipatory nausea and vomiting. Support Care Cancer 19: $1533-1538,2011$.

5. Roila F, Herrstedt J, Aapro M, et al: Guideline update for MASCC and ESMO in the prevention of chemotherapy- and radiotherapyinduced nausea and vomiting: results of the Perugia consensus conference. Ann Oncol 21: S232-S243, 2010

6. Basch E, Prestrud AA, Hesketh PJ, et al: Antiemetics: American society of clinical oncology clinical practice guideline update. J Clin Oncol 29: 4189-4198, 2011.

7. National Comprehensive Cancer Network (NCCN): Clinical Practice Guidelines in Oncology. Antiemesis. Version I, 2011. (Available at: http://www.nccn.org/professionals/physician_gls/ pdf/antiemesis.pdf). Accessed April 12, 2012.

8. de Wit R, Herrstedt J, Rapoport B, et al: Addition of the oral $\mathrm{NK}_{1}$ antagonist aprepitant to standard antiemetics provides protection against nausea and vomiting during multiple cycles of cisplatinbased chemotherapy. J Clin Oncol 21: 4105-4111, 2003.

9. Grunberg S, Chua D, Maru A, et al: Single-dose fosaprepitant for the prevention of chemotherapy-induced nausea and vomiting associated with cisplatin therapy: randomized, double-blind study protocol - EASE. J Clin Oncol 29: 1495-1501, 2011.

10. Hesketh PJ, Grunberg SM, Gralla RJ, et al: The oral neurokinin-1 antagonist aprepitant for the prevention of chemotherapy-induced nausea and vomiting: a multinational, randomized, double-blind, placebo-controlled trial in patients receiving high-dose cisplatin - the Aprepitant Protocol 052 Study Group. J Clin Oncol 21: 4112-4119, 2003

11. Rapoport BL, Jordan K, Boice JA, et al: Aprepitant for the prevention of chemotherapy-induced nausea and vomiting associated with a broad range of moderately emetogenic chemotherapies and tumor types: a randomized, double-blind study. Support Care Cancer 18: 423-431, 2010.

12. Hesketh PJ, Grunberg SM, Herrstedt J, et al: Combined data from two phase III trials of the $\mathrm{NK}_{1}$ antagonist aprepitant plus a $5 \mathrm{HT}_{3}$ antagonist and a corticosteroid for prevention of chemotherapyinduced nausea and vomiting: effect of gender on treatment response. Support Care Cancer 14: 354-360, 2006.

13. von der Maase H, Hansen SW, Roberts JT, et al: Gemcitabine and cisplatin versus methotrexate, vinblastine, doxorubicin, and cisplatin in advanced or metastatic bladder cancer: results of a large, randomized, multinational, multicenter, phase III study. J Clin Oncol 18: 3068-3077, 2000.
14. Tobinai K, Ogura M, Itoh K, et al: Randomized phase II study of concurrent and sequential combinations of rituximab plus $\mathrm{CHOP}$ (cyclophosphamide, doxorubicin, vincristine and prednisolone) chemotherapy in untreated indolent B-cell non-Hodgkin lymphoma: 7-year follow-up results. Cancer Sci 101: 2579-2585, 2010.

15. Piccart MJ, Di Leo A, Beauduin M, et al: Phase III trial comparing two dose levels of epirubicin combined with cyclophosphamide with cyclophosphamide, methotrexate, and fluorouracil in nodepositive breast cancer. J Clin Oncol 19: 3103-3110, 2001.

16. Scagliotti GV, Parikh P, von Pawel J, et al: Phase III study comparing cisplatin plus gemcitabine with cisplatin plus pemetrexed in chemotherapy-naive patients with advanced-stage non-small-cell lung cancer. J Clin Oncol 26: 3543-3551, 2008.

17. Ohe Y, Ohashi Y, Kubota K, et al: Randomized phase III study of cisplatin plus irinotecan versus carboplatin plus paclitaxel, cisplatin plus gemcitabine, and cisplatin plus vinorelbine for advanced non-small-cell lung cancer: Four-Arm Cooperative Study in Japan. Ann Oncol 18: 317-323, 2007.

18. Ogura M, Itoh K, Kinoshita T, et al: Phase II study of ABVd therapy for newly diagnosed clinical stage II-IV Hodgkin lymphoma: Japan Clinical Oncology Group study (JCOG 9305). Int J Hematol 92: 713-724, 2010.

19. Lefebvre JL, Chevalier D, Luboinski B, Kirkpatrick A, Collette L and Sahmoud T: Larynx preservation in pyriform sinus cancer: preliminary results of a European Organization for Research and Treatment of Cancer phase III trial. EORTC Head and Neck Cancer Cooperative Group. J Natl Cancer Inst 88: 890-899, 1996.

20. de Wit R, Roberts JT, Wilkinson PM, et al: Equivalence of three or four cycles of bleomycin, etoposide, and cisplatin chemotherapy and of a 3- or 5-day schedule in good-prognosis germ cell cancer: a randomized study of the European Organization for Research and Treatment of Cancer Genitourinary Tract Cancer Cooperative Group and the Medical Research Council. J Clin Oncol 19: 1629-1640, 2001.

21. Vitolo U, Chiappella A, Ferreri AJ, et al: First-line treatment for primary testicular diffuse large B-cell lymphoma with rituximab-CHOP, CNS prophylaxis, and contralateral testis irradiation: final results of an international phase II trial. J Clin Oncol 29: 2766-2772, 2011.

22. Dogliotti L, Carteni G, Siena S, et al: Gemcitabine plus cisplatin versus gemcitabine plus carboplatin as first-line chemotherapy in advanced transitional cell carcinoma of the urothelium: results of a randomized phase 2 trial. Eur Urol 52: 134-141, 2007.

23. Warr DG, Hesketh PJ, Gralla RJ, et al: Efficacy and tolerability of aprepitant for the prevention of chemotherapy-induced nausea and vomiting in patients with breast cancer after moderately emetogenic chemotherapy. J Clin Oncol 23: 2822-2830, 2005.

24. Poli-Bigelli S, Rodrigues-Pereira J, Carides AD, et al: Addition of the neurokinin 1 receptor antagonist aprepitant to standard antiemetic therapy improves control of chemotherapy-induced nausea and vomiting. Results from a randomized, double-blind, placebo-controlled trial in Latin America. Cancer 97: 3090-3098, 2003.

25. Chawla SP, Grunberg SM, Gralla RJ, et al: Establishing the dose of the oral $\mathrm{NK}_{1}$ antagonist aprepitant for the prevention of chemotherapy-induced nausea and vomiting. Cancer 97: 2290-2300, 2003.

26. Jordan K, Kinitz I, Voigt W, Behlendorf T, Wolf HH and Schmoll HJ: Safety and efficacy of a triple antiemetic combination with the NK-1 antagonist aprepitant in highly and moderately emetogenic multiple-day chemotherapy. Eur J Cancer 45: 1184-1187, 2009. 